\section{JTI}

JOURNAL OF

TRAUMA AND INJURY

Received: August 29, 2019

Revised: November 7, 2019

Accepted: December 2, 2019

\section{Correspondence to}

Young Hoon Sul, M.D., Ph.D.

Department of Trauma Surgery, College of Medicine, Chungbuk National University,

1 Chungdae-ro, Seowon-gu, Cheongju

28644, Korea

Tel: +82-43-269-7847

Fax: +82-43-269-7810

E-mail:ssulyh@gmail.com

\title{
Chronic Traumatic Glass Foreign Body Removal from the Lung through a Direct Parenchymal Incision
}

\author{
Su Young Yoon, M.D. ${ }^{1}$, Si Wook Kim, M.D., Ph.D. ${ }^{1}$, Jin Suk Lee, M.D. ${ }^{2}$, \\ Jin Young Lee, M.D. ${ }^{2}$, Jin Bong Ye, M.D. ${ }^{2}$, Se Heon Kim, M.D. ${ }^{2}$, \\ Young Hoon Sul, M.D., Ph.D., ${ }^{2,3}$ \\ ${ }^{1}$ Department of Thoracic and Cardiovascular Surgery, Chungbuk National University \\ Hospital, Cheongju, Korea \\ ${ }^{2}$ Department of Trauma Surgery, Chungbuk National University Hospital, Cheongju, \\ Korea \\ ${ }^{3}$ Department of Trauma Surgery, College of Medicine, Chungbuk National University, \\ Cheongju, Korea
}

Traumatic intrapulmonary glass foreign bodies that are missed on an initial examination can migrate and lead to severe complications. Here, we present a rare case of a traumatic intrapulmonary glass foreign body surgically removed by a direct pulmonary incision, which preserved the pulmonary parenchyma and avoided severe complications caused by migration.

Keywords: Wounds and injuries; Foreign bodies; Surgical procedures, Operative; Thoracotomy

\section{INTRODUCTION}

Traumatic foreign bodies are usually diagnosed and treated adequately, but they can be missed due to their radiolucent characteristics, location, and negligence. Some foreign bodies result in immediate inflammatory complications, whereas others can remain asymptomatic for a long time [1]. Organic materials such as wood can trigger an acute severe inflammatory reaction; in contrast, inorganic materials such as glass shards may remain asymptomatic if there is no significant physical injury [2]. We present a case of a chronic intrapulmonary foreign body identified as a glass shard, which was successfully removed by direct pulmonary incision through a minimal thoracotomy rather than via lung resection, as described in other reports. (http://creativecommons.org/licenses/by-nc/4.0/) which permits unrestricted noncommercial use, distribution, and reproduction in any medium, provided the original work is properly cited. 


\section{CASE REPORT}

A 50-year-old male farmer presented with 6-month history of cough and sputum. Ten years previously, he slipped in the bathroom and broke a window, resulting in a small laceration on his right upper back. The injury was sutured at a nearby hospital. Over several months, he developed a negligible cough with mild white sputum and no other symptoms. He began to experience a large amount of whitish sputum, and shortly thereafter was admitted to the hospital for evaluation. On physical examination, a linear small scar was observed on his right upper back. Chest auscultation revealed rhonchi sounds in the upper pulmonary fields. The laboratory findings showed a hemoglobin concentration of $13.1 \mathrm{~g} / \mathrm{dL}$, a white blood cell count of $10.2 \times 10^{3} / \mathrm{mm}^{3}$, and a platelet count of $11.6 \times 10^{3} / \mu \mathrm{L}$. On chest radiography, a suspicious intrapulmonary for- eign body mimicking a pulmonary vascular shadow was identified (Fig. 1A). Chest computed tomography (CT) revealed a sharp-edged foreign body surrounded by a cavity in the right lower pulmonary lobe (Fig. 1B, C). Considering the location of the old scar and a localized pleural thickening on chest CT, it might have passed through the chest wall. Fortunately, the cavitary lesion communicated with a small bronchiole, which acted as a drain. Due to severe pleural adhesion, video-assisted thoracoscopic surgery with a minimal thoracotomy was performed, and the foreign body was successfully removed through a direct parenchymal incision. The small fibrotic cyst was obliterated by suturing. The foreign body was identified as a triangular, sharp glass shard $4 \mathrm{~cm}$ in length (Fig. 2). His symptoms immediately disappeared, and he was discharged 6 days postoperatively.
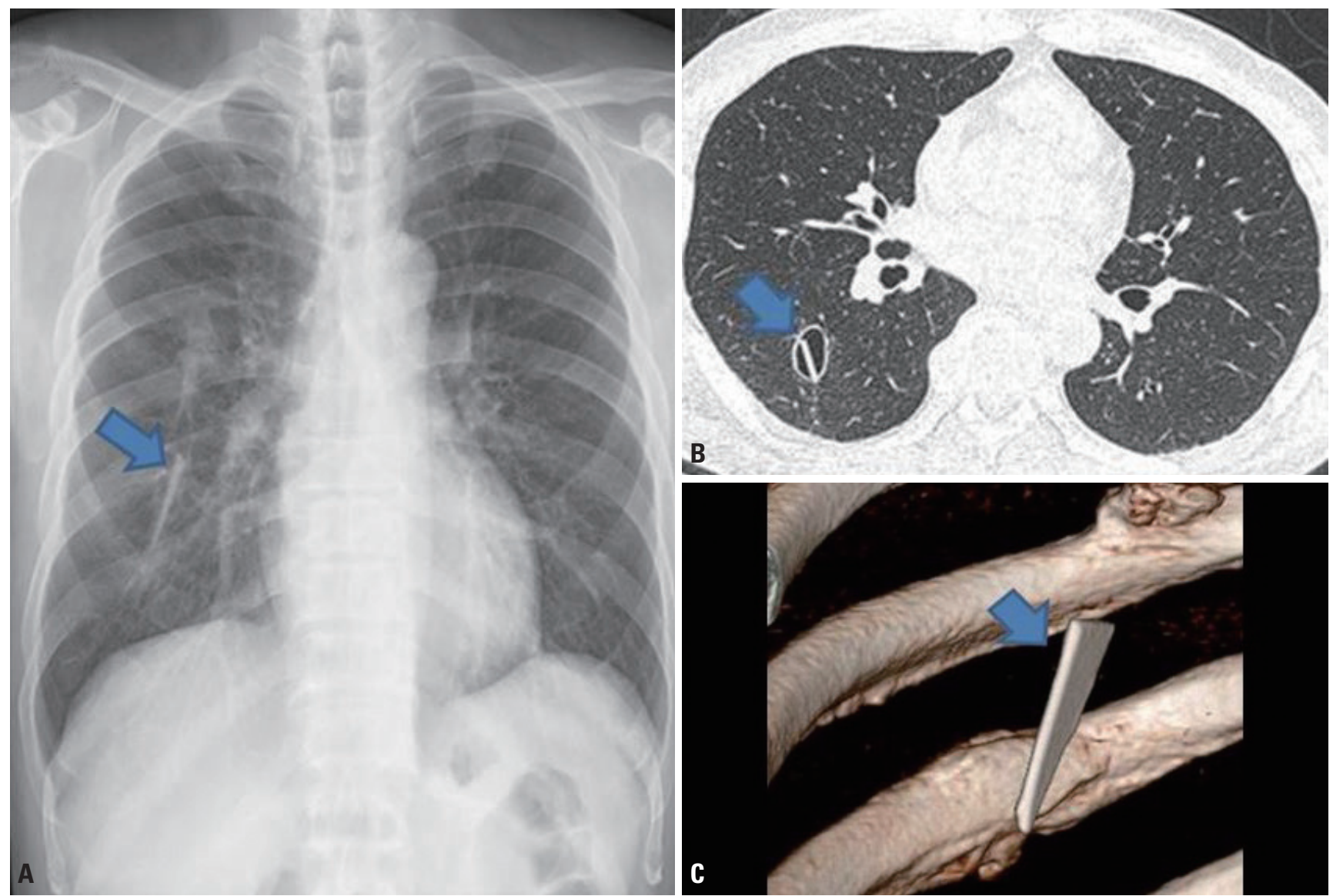

Fig. 1. (A) Chest radiography showed a linear, sharp opacity in right lower lobe. (B) Computed tomography (CT) showed a fibrotic cyst surrounding the foreign body without inflammatory parenchymal change. (C) 3D CT demonstrated the intrapulmonary foreign body. 


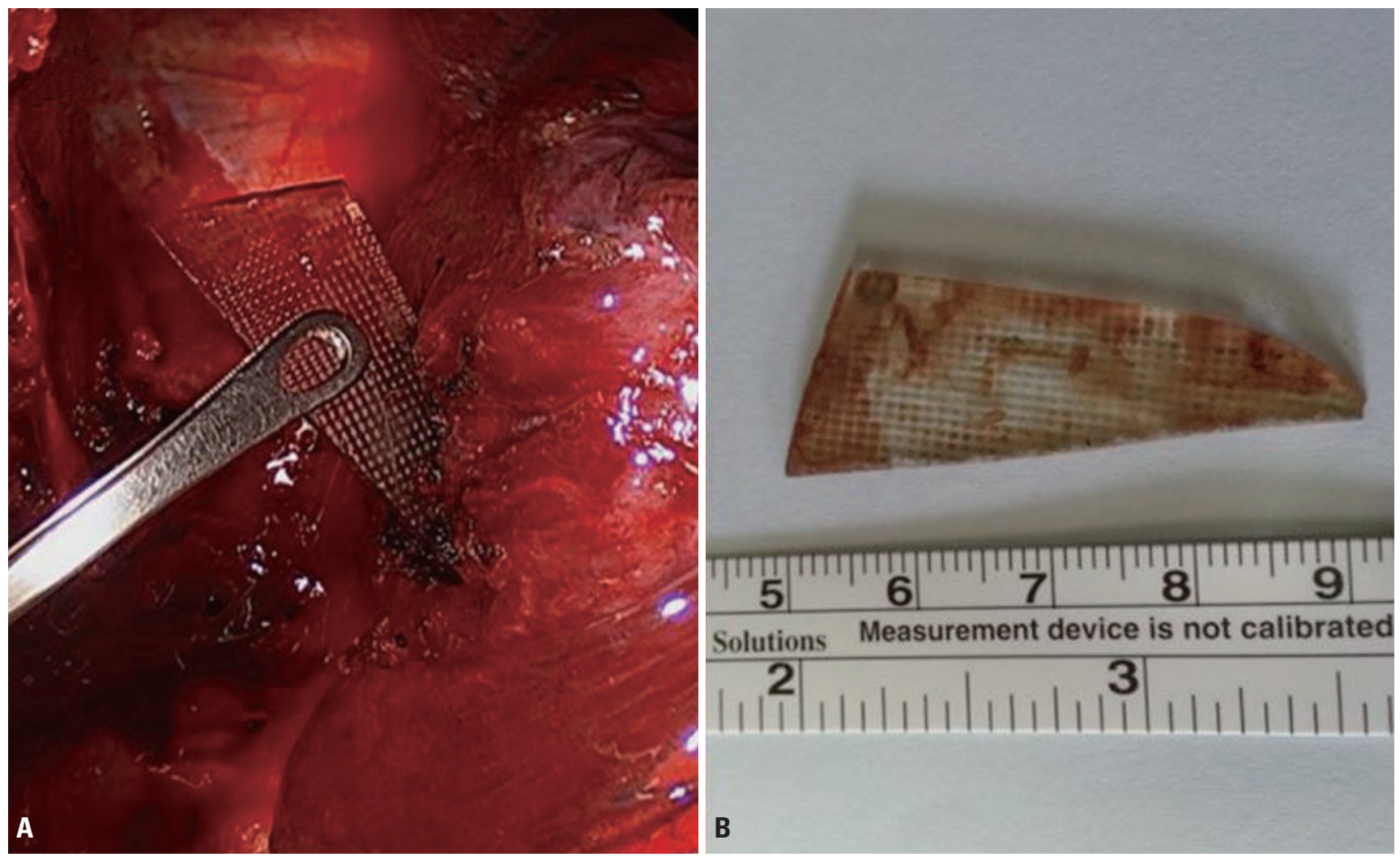

Fig. 2. (A) The intraoperative view showed the intrapulmonary foreign body removed through a direct parenchymal incision. (B) The removed intrapulmonary foreign body.

\section{DISCUSSION}

Glass injuries account for approximately $13 \%$ of all traumatic wounds. Glass is the most frequently retained foreign body that is often missed in the initial diagnosis [1]. For patients with penetrating thoracic wounds, early diagnosis is crucial because of the potential for life-threatening complications.

Chest radiography is recommended to detect potential hemothorax, pneumothorax, and retained foreign bodies [3]. Radiography is an inexpensive and effective modality for imaging glass foreign bodies, and nearly all glass debris can be detected, except when obscured by bone. On radiography, glass fragments measuring $2 \mathrm{~mm}$ or more in length can be detected with approximately $100 \%$ accuracy [3]. In the present case, the patient could not remember whether radiography was performed following the initial injury. Subsequent imaging during routine examinations may have missed the foreign body due to its vague location, which could have been misinterpreted as a pulmonary vascular shadow, and the low resolution of radiography. Fortunately, serious complications, such as hemothorax, pneumothorax, and pneumonia did not occur. Chronic intrapulmonary foreign bodies frequently become enclosed by cystic fibrosis [4,5].

Various foreign bodies have been described in previous reports. The severity of the inflammatory reaction depends on the specific material. In addition, the shape and size of the foreign body affect the severity of damage caused by mechanical injury during migration. In some cases, elusive glass foreign bodies embedded in the musculoskeletal system that cause little functional deficit may be left in place because surgery may result in significant injury during removal of the object $[2,3]$. However, in the lung, large glass foreign bodies must be removed because of the risk of severe vascular injury if they migrate. A variety of intrapulmonary foreign bodies have been removed by pulmonary resection, regardless of whether the 
parenchyma was normal or reversibly injured [5]. In this patient, we performed a direct parenchymal incision and removed the chronic intrapulmonary foreign body and its surrounding minimal cystic fibrosis. In this case, foreign body measured about $4 \mathrm{~cm}$, so it was possible to find it by hand, if there is a small foreign body in the lung parenchyma, CT-guided methylene blue staining or CT-guided hook wire localization is commonly used, and C-arms are sometimes used in the operating room.

Intrapulmonary glass foreign bodies that are missed on the initial examination can migrate and lead to severe complications. Therefore, these objects must be removed at the initial diagnosis if possible. If the foreign body comprises inorganic material such as glass and is palpable, then it can be removed by simple direct pulmonary incision, which preserves the pulmonary parenchyma and potentially avoids significant complications caused by migration.

\section{REFERENCES}

1. Kaiser CW, Slowick T, Spurling KP, Friedman S. Retained foreign bodies. J Trauma 1997;43:107-11.

2. Ozsarac M, Demircan A, Sener S. Glass foreign body in soft tissue: possibility of high morbidity due to delayed migration. J Emerg Med 2011;41:e125-8.

3. Orlinsky M, Bright AA. The utility of routine X-rays in all glasscaused wounds. Am J Emerg Med 2006;24:233-6.

4. Yurdakul AS, Hoca NT, Gökcek A, Cimen F, Cakaloglu A, Atikcan S, et al. An interesting cause of recurrent haemoptysis: haemoptysis 7 years after a foreign body penetrated the lung parenchyma and aorta. Respirology 2005;10:229-32.

5. Miura H, Taira O, Hiraguri S, Hirata T, Kato H. Successful surgical removal of an intrapulmonary aberrant needle under fluoroscopic guidance: report of a case. Surg Today 2001;31:55-8. 